\title{
Globe
}

Revue internationale d'études québécoises

Annie Becquer, et al. : Femme, j'écris ton nom... Guide d'aide à la féminisation des noms de métiers, titres, grades et fonctions, Paris, La Documentation française, 1999, 124 p.

\section{Noëlle Guilloton}

Volume 3, numéro 2, 2000

URI : https://id.erudit.org/iderudit/1000591ar

DOI : https://doi.org/10.7202/1000591ar

Aller au sommaire du numéro

Éditeur(s)

Globe, Revue internationale d'études québécoises

ISSN

1481-5869 (imprimé)

1923-8231 (numérique)

Découvrir la revue

Citer ce compte rendu

Guilloton, N. (2000). Compte rendu de [Annie Becquer, et al. : Femme, j'écris ton nom... Guide d'aide à la féminisation des noms de métiers, titres, grades et

fonctions, Paris, La Documentation française, 1999, 124 p.] Globe, 3(2), 194-198. https://doi.org/10.7202/1000591ar d'utilisation que vous pouvez consulter en ligne.

https://apropos.erudit.org/fr/usagers/politique-dutilisation/ 
dans la théorie s'attaque de front au problème épineux de "l'identité de l'auteur ". Cela aurait été d'autant plus intéressant que cette question se trouve au centre d'une part importante de la critique littéraire actuelle, des gay studies aux black studies en passant par les aboriginal studies, sans parler, évidemment, des women studies. La controverse n'est donc pas près de s'éteindre...

Élisabeth Rousseau

Collège André-Grasset

\section{Annie Becquer, et al.}

Femme, j'écris ton nom... Guide d'aide à la féminisation des noms de métiers, titres, grades et fonctions

Paris, La Documentation française, 1999, 124 p.

"Le français dispose de ressources morphosyntaxiques qui permettent une féminisation conforme au génie de la langue dans quasiment tous les cas. "Cette phrase qui figure en quatrième de couverture de Femme, j'écris ton nom traduit bien la position que le Québec a prise et prônée depuis une vingtaine d'années quant à la féminisation des appellations de personnes. On sait qu'effectivement nous avons fait œuvre de pionniers et de pionnières en la matière. La Suisse a emboîté le pas quelques années plus tard, puis la Belgique au début des années ' 90 . Mais la France restait à la traîne, malgré une première circulaire administrative publiée en 1986, énonçant des règles de formation des féminins et encourageant leur emploi... C'est pourquoi ce guide rédigé par l'Institut national de la langue française (Inalf) de France vient à point. Préfacé par Lionel Jospin, le premier ministre en personne, il répond à une demande que ce promoteur de la parité et de l'égalité avait lui-même formulée.

Voilà un petit ouvrage de vulgarisation, accessible à tous ceux et celles que la langue intéresse, qui résume de façon fort intéressante l'historique de la question, présente les règles de féminisation, aborde les objections et les difficultés rencontrées, et expose le mode de constitution de la liste des substantifs féminins proposés. Cette liste 


\section{RECENSIONS}

de plus de 2000 noms de métiers, grades, titres et fonctions au masculin et au féminin complète le tout. Le titre peut toutefois indisposer : s'agit-il d'une envolée poétique inspirée par le "J'écris ton nom, liberté! " d'Éluard, ou d'une résolution autoritaire aux relents paternalistes voire machistes? Signalons en passant que le guide est écrit conformément aux Rectifications de l'orthographe du Conseil supérieur de la langue française, publiées en 1990. On remarque donc notamment l'omission de l'accent circonflexe sur le $\mathrm{i}$ et le $\mathrm{u}$ (bucheron, maitre), l'accentuation des mots empruntés (imprésario) et la soudure des mots composés d'éléments nominaux et adjectivaux (hautecontre, sagefemme). C'est l'occasion de constater que ces modifications ne sont pas si curieuses, et que les occurrences des mots ainsi modifiés ne sont pas si nombreuses!

Cet ouvrage est divisé en quatre grandes sections respectivement consacrées à la féminisation dans l'histoire, aux règles grammaticales touchées par cette question, aux arguments développés pour et contre la féminisation, et à la liste de métiers, titres, grades et fonctions. La partie historique est d'autant plus importante que l'on a parfois tendance à négliger cette dimension des phénomènes linguistiques. En matière de féminisation, l'évolution de l'usage procure un éclairage intéressant. Ainsi, le guide rappelle que le latin offrait suffixes masculins et féminins, et que la langue française du XIIe au XVIe siècles a créé quantité de féminins. Pour rechercher ces mots, l'INALF a mis à contribution avec profit sa base textuelle Frantext sur le moyen français. Celle qui porte sur le français moderne a permis, quant à elle, de repérer des attestations de féminins " en progression indiscutable ». Les règles grammaticales, pour leur part - elles n'ont rien de nouveau pour nous -, traitent successivement de l'emploi du déterminant féminin, des noms qui se terminent au masculin par une voyelle, de ceux qui se terminent par une consonne, des abréviations et des sigles, des mots empruntés à une langue étrangère, de quelques cas particuliers et de l'accord dans les dénominations composées et complexes. Les règles qui retiennent le plus notre attention sont évidemment celles des noms se terminant par -eur et des noms se terminant par -teur. Ce sont effectivement celles qui suscitent le plus d'interrogations chez les locuteurs et locutrices, et sur lesquelles certaines divergences et concurrences se 
manifestent dans la francophonie. Ainsi, pour les noms en -eur, «lorsqu'il n'existe pas de verbe correspondant au nom ou que le verbe n'est pas en rapport sémantique direct ", le guide donne le choix entre l'emploi épicène et l'ajout d'un e final (une ingénieur ou une ingénieure, par exemple). Il en va de même pour auteur, docteur et pasteur. Dans la liste des appellations, ces féminins sont écrits avec un $e$ final entre parenthèses, indiquant une possible double lecture.

Ce que Femme, j'écris ton nom établit - ou, plutôt, rappelle - de façon convaincante, c'est que les obstacles à la féminisation sont essentiellement d'ordre psychologique et socioculturel, et non pas d'ordre linguistique. Le chapitre consacré aux « objections et difficultés " est donc particulièrement bienvenu. Il traite successivement des arguments linguistiques comme l'homonymie et l'euphonie auxquels on fait souvent appel à des fins peu scientifiques, puis de la dévalorisation, argument éminemment psychosocial. Le mot féminin désigne une machine? Tailleur, secrétaire, avocat et bien d'autres mots masculins désignent l'un un vêtement, l'autre un meuble, l'autre un fruit... sans faire sourciller quiconque. L'homonymie et la polysémie sont des faits de langue courants : le contexte suffit à lever les ambiguités. Quant à l'euphonie, pourquoi servirait-elle à rejeter des mots français féminins bien formés, alors qu'on y semble peu sensible lorsqu'il s'agit d'emprunts beaucoup plus difficiles à prononcer (discjockey, par exemple)? La réponse à cette question ("la néologie est dissonante quand elle est dérangeante ") mène tout droit à l'argument de la dévalorisation du féminin. En effet, assez souvent, les femmes elles-mêmes sont réticentes à porter un titre féminin, le jugeant dépréciatif. Le guide explique la cause de ce sentiment bien présent en France. Par les exemples qu'il donne, les contextes qu'il cite, les rapprochements qu'il fait, l'ouvrage atteste qu'il s'agit bien là de la manifestation d'un " complexe d'infériorité », comme l'écrivait déjà le linguiste Albert Dauzat en 1955. Voulant faire appel au sentiment - voire à la raison - linguistique des locuteurs et locutrices, certains avancent l'argument du neutre, ou de la neutralité du genre non marqué, le masculin. L'ouvrage rappelle en quoi cet argument est fallacieux : il n'y a que deux genres pour les noms en français et, pour les animés désignant des humains, le genre suit le sexe, à de rares exceptions près, même si certains 
souhaiteraient peut-être généraliser celles-ci pour justifier un recours au masculin dans tous les cas.

La "question du générique " nous laisse toutefois sur notre faim, car elle risque de fermer la porte à toute tentative de féminisation des textes. On sent que le guide a dû tenir compte du Rapport sur la féminisation des noms de métier, fonction, grade ou titre publié sous l'égide de la Commission générale de terminologie de France en 1998. Ce rapport, peu favorable à la féminisation, faisait la part belle à la langue juridique et réglementaire - voire administrative? - et, de façon plutôt obscure et artificielle, insistait sur la nécessité de distinguer le nom de la fonction (pour laquelle seul conviendrait le masculin "générique») de celui de sa titulaire. Le guide semble même étendre l'usage d'un tel masculin aux offres de recrutement. $\mathrm{Ce}$ qui surprend alors, c'est que le titre de l'ouvrage inclut bien la féminisation des noms de fonctions! On est probablement ici devant un conflit d'autorités, et on se heurte au conservatisme d'un usage qui est qualifié de " républicain "... Quant à ce qui est présenté comme " certaines habitudes francophones" du genre: "Recrutement d'un ou une attaché(e) parlementaire ", il aurait été pertinent de préciser que le procédé d'écriture qui met le féminin entre parenthèses ne figure dans aucun guide québécois, belge ou suisse. À moins que ces " habitudes » que l'ouvrage désapprouve soient celles de féminiser les fonctions et les offres d'emploi, ce que nous continuons de trouver parfaitement justifié?

Enfin, une longue liste de 2200 féminins, dressée selon des critères bien définis, propose des féminins, classiques et néologiques, accompagnés du numéro de la règle selon laquelle ils sont formés. En marge, s'inspirant de la présentation de la liste belge Mettre au féminin, figurent des notes signalant soit une variante morphologique, soit une précision étymologique, soit une mention de l'usage au Québec, en Suisse ou en Belgique, soit un rappel du statut officiel du terme. Une publication comme Femme, j'écris ton nom était attendue depuis longtemps dans la francophonie. Ce n'est pas parce le Québec, la Suisse ou la Belgique en avaient besoin - chacun avait déjà son guide, fort bien reçu - mais parce qu'il était temps que la France réponde aussi à cette évolution des besoins linguistiques, et que des 
linguistes à l'esprit ouvert fassent contrepoids à la résistance têtue et quelque peu empreinte de mauvaise foi - de membres de l'Académie française. Le guide est utile et contribuera sans nul doute à renforcer la sécurité linguistique des francophones en matière de féminisation. On peut simplement regretter le peu de reconnaissance que ses responsables accordent aux qualités innovatrices des travaux du Québec en la matière...

\section{Noëlle Guilloton en coll. avec Pierrette Vachon-L'Heureux Office de la langue française}

\section{Andrée Lévesque}

Scènes de la vie en rouge

L'époque de Jeanne Corbin, 1906-1944

Montréal, Les Éditions du remue-ménage, 1999, 309 p.

L'idée est géniale : raconter aux jeunes militantes féministes d'aujourd'hui la vie d'une jeune communiste canadienne d'entre les deux guerres. Celle de Jeanne Corbin, une francophone issue des classes populaires, est un choix logique. Néanmoins, le défi est de taille, car il n'existe à son sujet que de rares sources documentaires. Née en France et élevée dans une ferme en Alberta, Jeanne Corbin fut une jeune militante communiste à Edmonton, à Toronto et à Montréal, avant d'œuvrer pendant dix ans à Timmins, une ville minière du nord de l'Ontario. Suivie de près par la Gendarmie royale depuis son adolescence, Corbin fut arrêtée à Toronto, harcelée à Montréal et incarcérée en Abitibi. Son bref séjour en prison, pour avoir suscité une émeute chez des bûcherons, porte atteinte à sa santé. À l'automne 1942, elle entre au sanatorium de London, en Ontario. Dix-huit mois plus tard, cette " héroïne du parti ", toujours confiante de la victoire prochaine du peuple, meurt. Elle n'avait que 38 ans.

Cette militante de la base fut toujours parrainée, sinon protégée, par des membres influents du parti. $\grave{A} 16$ ans, elle fait la connaissance 